05

\title{
Усиление пьезоэлектрических и диэлектрических свойств и макроскопическая релаксация зарядового и полевого отклика в 0-3 композитах „керамика-поры“: теория и эксперимент
}

\author{
(C) Г.С. Радченко, ${ }^{1}$ А.В. Скрылев, ${ }^{2}$ А.Ю. Малыхин, ${ }^{2}$ А.А. Панич ${ }^{2}$ \\ ${ }^{1}$ Южный федеральный университет, \\ 344022 Ростов-на-Дону, Россия \\ ${ }^{2}$ Южный фредеральный университет, \\ Институт высоких технологий и пьезотехники, \\ 344090 Ростов-на-Дону, Россия \\ e-mail: grig1980@mail.ru
}

(Поступило в Редакцию 3 июля 2017 г.)

\begin{abstract}
Теоретически и экспериментально описаны диэлектрические и пьезоэлектрические свойства композитов, обладающих связностью 0-3 на основе керамических составов типа цирконат-титанат свинца. На основе предлагаемой модели теоретически и экспериментально определены частотные зависимости диэлектрического и пьезоэлектрического отклика. Рассмотрено влияние максвелл-вагнеровской релаксации и приближения эффективных параметров на физические свойства неупорядоченных объектов типа „статистическая смесь“. Построены функции распределения времен межкомпонентной релаксации композитной системы „пьезокерамика-поры“ и концентрационные зависимости эффективных полевых откликов. Сделаны сравнения полученных теоретических результатов и проведенного эксперимента.
\end{abstract}

DOI: 10.21883/JTF.2018.02.45408.2455

\section{Введение}

Пористые керамические композитные материалы в настоящее время вызывают особый интерес. Это связано с необычными свойствами подобных материалов как под действием электрических, так и под действием волновых механических полей направленного звука [1-14]. В частности, уникальным свойством пористых керамических композитов является сохранение и даже увеличение пьезоэлектрических свойств при высоких концентрациях воздушных пор [5,6]. Данное обстоятельство приводит к повышенной объемной пьезочувствительности таких составов, что делает их перспективными для многих технических применений. Между тем при описании таких составов многие проблемы как теоретического, так и экспериментального характера остаются нерешенными и даже неупомянутыми в литературе. Среди них можно отметить межфазное накопление заряда на поверхностях раздела „поры-керамика“ и связанные с этим как релаксацию, так и частотную зависимости эффективных констант. Необходимым условием измерения эффективных параметров композита является режим термодинамического равновесия и выполнение адиабатического приближения, что при комнатной температуре и нормальных условиях выполнено в подавляющем числе случаев. В этом случае к таким средам для теоретического описания вполне применимы все уравнения состояния пьезоактивных сред, и выбор той или иной формы их записи диктуется конкретной технической направленностью и целью расчетов, измерений или предполагаемым реше- нием технической задачи. При измерении, например, квазистатического пьезоэлектрического отклика очень часто пренебрегают его частотной зависимостью, что является совершенно неоправданным. Известный факт роста диэлектрической проницаемости (ДП) с уменьшением частоты внешнего воздействия на низких и инфранизких частотах $[1,2]$ объясняется именно возникновением значительного объемного заряда. Между тем, как видно из основных уравнений пьезоэлектричества, частотная зависимость ДП влечет за собой аналогичную зависимость и фазовый сдвиг абсолютно всех констант, в том числе пьезоэлектрических, диэлектрических и упругих $[1,2,13,14]$. Самому вопросу возникновения токов проводимости при квазистатических измерениях и связанного с этим межслойного накопления заряда на границах пор в литературе уделено явно недостаточное внимание, что и стимулировало настоящую работу.

\section{Теоретическое исследование}

В настоящее время все методы описания гетерофазных, в частности, двухфазных композитов делятся на два типа: описание матричных систем и систем типа „статическая смесь“. В случае матричного композита один из компонентов все время образует связную матрицу, в которой находятся включения другого компонента. Система при этом остается структурно асимметричной, и возрастание концентрации включений теоретически не должно менять структуру материала. Практически это является малодостижимым уже при концентрациях 
включений порядка 40-50\%. В таких системах при расчетах по матричным методам теоретически невозможны перколяционные переходы типа, например, „металлдиэлектрик“, или „жесткость-податливость“. Это обстоятельство в ряде случаев находится в резком противоречии с экспериментальными данными, которые ясно указывают на наличие таких переходов в реальных керамиках и о резких аномалиях физических свойств около соответствующих им концентраций компонентов. Сложный композитный материал может моделироваться статистической смесью сфер или сфероидов. Конкретное аспектное отношение, а также ориентация составляющих композит сфероидальных частиц как факторы влияния могут быть исключены из рассмотрения при переходе к правильным сферам. Именно это упрощение по сравнению с важнейшей работой [10] было сделано Алешиным в [5] для анизотропной керамики и в [6] для композитов. И поэтому основной проблемой для расчета композитных сред и взаимодействия сферического включения со средой является вычисление тензора Эшелби из одноименной микромеханической модели [5,6,8-10].

Для теоретических расчетов в табл. 1,2 мы применяем модифицированный метод [5,6] для описания смеси двух трансверсально изотропных керамик $[5,6,8-10]$, и в качестве предельного случая рассматриваем пористый композит 0-3 на основе керамики цитанат-титанат свинца (ЦТС) по [6]. Поры предполагаются обладающими нулевой упругой жесткостью (или бесконечной податливостью [6]). Метод [6] также дополнен нами с учетом сквозной проводимости и межслойной поляризации по моделям [1,2]. Механически свободная ДП представляется нами как $\varepsilon_{33}^{\sigma}=\left.\varepsilon_{33}^{\sigma}\right|_{\omega \rightarrow \infty}+i \frac{\gamma}{\omega}$, где $\gamma$ есть сквозная проводимость чистой керамики в направлении поляризации. Здесь $\varepsilon$ есть ДП, $\omega-$ круговая (циклическая) частота. Случай пористого композита сравнивается с проведенным экспериментом на серии четырех частот, которые охватывают практически всю релаксационную максвелл-вагнеровскую область $[1,2,13,14]$. Десять исходных констант [5-7] керамики ЦТС брались из ОСТ 11 0444-87 [7] для составов ЦТС-36 и ЦТБС-3. На выходе программа выдавала десять в общем случае комплексных констант [6], что позволяло теоретически рассчитывать их абсолютные значения и фазовые параметры. Теория метода достаточно полно изложена в $[5,6]$ и мы не будем здесь выписывать соответствующие достаточно громоздкие формулы, полностью следуя им при вычислениях. Важно отметить, что при корректном следовании теории $[5,6]$ возможно составить итерационную программу для расчета десяти важнейших констант пористого композита, что и было сделано нами в настоящей работе.

\section{Экспериментальное исследование}

Для изготовления пьезокерамических материалов системы ЦТС был использован метод твердофазных реак- ций. Из порошков оксидов и солей металлов, входящих в состав комплексного соединения пьезокерамического материала, изготавливалась шихта путем смешивания в мельнице центробежно-планетарного типа. Далее она подвергалась температурной обработке, в процессе которой происходил синтез материала. С целью обеспечения сохранности стехиометрического состава пьезокерамического материала, в качестве емкости для синтеза использовались плотно закрывающиеся корундовые тигли. Затем полученный синтезированный порошок измельчался. Из синтезированного материала были изготовлены пробники цилиндрической формы, предназначенные для контроля электрофизических, пьезоэлектрических и механических характеристик. Для получения элементов с заданной степенью пористости был проведен эксперимент, позволяющий определить зависимость конечной пористости спеченного образца от ее теоретических начальных значений. В ходе эксперимента использовался порообразователь на основе солей аммония. Внутрь синтезированного пьезокерамического материала непосредственно перед формовкой вводилось определенное количество порообразователя и затем изделие последовательно подвергалось формовке, сушке и обжигу. Температура обжига пьезокерамических элементов ЦТС составляла $1230-1300^{\circ} \mathrm{C}$, а температура разложения порообразователя равнялась $400-450^{\circ} \mathrm{C}$. При плавном нагреве частицы аммониевых солей образовывали газ и покидали сформированный каркас, образовывая поры с нулевой упругой жесткостью и $\varepsilon=1$. После проведения серии спеканий пьезокерамических элементов с различной степенью закладываемой пористости были получены пять серий образцов, которые отличались степенью пористости и размером электродов. Контроль степени пористости полученных элементов осуществлялся путем определения плотности и сравнения полученных величин с параметрами гомогенного образца. Плотность определялась как из геометрических расчетов, так и методом гидростатического взвешивания, что обеспечивало высокую дополнительную точность расчета пористости до сотых долей от объема (одного процента). Поляризация образцов осуществлялась как стандартным статическим, так и импульсным методом, что в итоге давало малые различия. Герметизация полиуретаном непосредственно перед измерениями проводилась посредством погружения. Измерения проводились на приборе „Паскаль-4“ при амплитуде звуковой волны $20 \mathrm{~Pa}$ Данный прибор создавал всестороннее сжатие образца низкочастотным волновым деформационным полем в специально отведенной для этой цели камере прибора. Индуцированный заряд и напряжение измерялись встроенным в прибор электрометром в зависимости от материала и частоты внешнего воздействия. Механическое напряжение четырех различных частот вызывало синфазные колебания электрического поля в образце, что позволило применить теоретическое описание, основанное на введении в рассмотрение комплексной ДП ([1-3] и выше). 
Таблица 1. Диэлектрические свойства пористых композитов 0-3 на основе системы ЦТС. Теория и эксперимент

\begin{tabular}{|c|c|c|c|c|}
\hline Номер состава & $\begin{array}{c}C^{*}, p F \\
(20 / 63 / 125) \mathrm{Hz}\end{array}$ & $\begin{array}{l}C_{\infty}, \mathrm{pF} \\
(1 \mathrm{kHz})\end{array}$ & $\begin{array}{c}\operatorname{tg} \delta, \% \\
(1 \mathrm{kHz})\end{array}$ & $\gamma, \mathrm{Sm} / \mathrm{m}$ \\
\hline $\begin{array}{c}1 \text { (эксперимент) } \\
1 \text { (теория) }\end{array}$ & $\begin{array}{l}243 / 240 / 238 \\
209 / 193 / 191 \\
\end{array}$ & $\begin{array}{l}222 \\
190 \\
\end{array}$ & $\begin{array}{l}1.4 \\
1.2 \\
\end{array}$ & $\begin{array}{l}6.1 \cdot 10^{-7} \\
6.1 \cdot 10^{-7}\end{array}$ \\
\hline $\begin{array}{c}2 \text { (эксперимент) } \\
2 \text { (теория) }\end{array}$ & $\begin{array}{l}223 / 220 / 218 \\
186 / 172 / 171 \\
\end{array}$ & $\begin{array}{l}202 \\
170 \\
\end{array}$ & $\begin{array}{l}1.5 \\
1.3 \\
\end{array}$ & $\begin{array}{l}6.1 \cdot 10^{-7} \\
6.1 \cdot 10^{-7}\end{array}$ \\
\hline $\begin{array}{c}3 \text { (эксперимент) } \\
3 \text { (теория) }\end{array}$ & $\begin{array}{l}320 / 319 / 318 \\
404 / 389 / 387 \\
\end{array}$ & $\begin{array}{l}298 \\
386 \\
\end{array}$ & $\begin{array}{c}1 \\
0.9 \\
\end{array}$ & $\begin{array}{l}7.7 \cdot 10^{-7} \\
7.7 \cdot 10^{-7}\end{array}$ \\
\hline $\begin{array}{c}4 \text { (эксперимент) } \\
4 \text { (теория) }\end{array}$ & $\begin{array}{l}1197 / 1187 / 1183 \\
1619 / 1555 / 1550 \\
\end{array}$ & $\begin{array}{l}1157 \\
1547 \\
\end{array}$ & $\begin{array}{c}1 \\
\underline{0.9} \\
\end{array}$ & $\begin{array}{l}7.7 \cdot 10^{-7} \\
7.7 \cdot 10^{-7}\end{array}$ \\
\hline $\begin{array}{c}5 \text { (эксперимент) } \\
5 \text { (теория) }\end{array}$ & $\begin{array}{l}294 / 291 / 288 \\
336 / 323 / 322 \\
\end{array}$ & $\begin{array}{l}273 \\
321 \\
\end{array}$ & $\begin{array}{c}1 \\
0.9 \\
\end{array}$ & $\begin{array}{l}7.7 \cdot 10^{-7} \\
7.7 \cdot 10^{-7}\end{array}$ \\
\hline
\end{tabular}

Для исследования существования межслойной поляризации и релаксации в полученных образцах пористых композитов 0-3 были выбраны:

1. ЦТС-36, содержание пор $37 \%$, размер образца $20 \times 20 \times 8.5 \mathrm{~mm}$,

2. ЦТС-36, содержание пор 40\%, размер образца $20 \times 20 \times 8.5 \mathrm{~mm}$,

3. ЦТБС-3, содержание пор 39\%, размер образца $20 \times 20 \times 8.5 \mathrm{~mm}$,

4. ЦТБС-3, содержание пор 39\%, размер образца $40 \times 40 \times 8.5 \mathrm{~mm}$,

5. ЦТБС-3, содержание пор 44\%, размер образца $20 \times 20 \times 8.5 \mathrm{~mm}$.

Зарядовый отклик $M_{q}$ определялся нами как $M_{q}=\left(2 d_{31}+d_{33}\right) S$, где $d_{31}$ и $d_{33}$ - эффективные пьезомодули пористого состава, $S$ - площадь электродов. Полевой отклик $M_{u}$ определялся как $M_{q} / C^{*}$, где $C^{*}$ - емкость в направлении исходной поляризации керамик ЦТС.

Удельная проводимость используемой нами керамики ЦТС изначально была неизвестна. Для ее определения и расчета частотной зависимости зарядового накопления на частоте $1 \mathrm{kHz}$ был измерен тангенс угла диэлектрических потерь. Сквозная электропроводность определялась по результатам экспериментальных данных по известной формуле (1) [14,15]:

$$
\gamma=\frac{\varepsilon_{\infty} \operatorname{tg} \delta}{1.8 \cdot 10^{10}} f
$$

Здесь $\varepsilon_{\infty}$ есть ДП, измеренная на $1 \mathrm{kHz}$ на правом краю частотной области (высокочастотная ДП), $\operatorname{tg} \delta$ есть экспериментально определенный тангенс угла диэлектрических потерь на $1 \mathrm{kHz}$, и $f=\omega / 6.28$, это частота внешнего воздействия, равная $1 \mathrm{kHz}$. Рассчитанная по формуле (1) проводимость с учтенной нами низкочастотной перколяционной поправкой на концентрацию пор представлена в последнем столбце табл. 1. Экспериментальные и теоретические результаты для ДП представлены в табл. 1, для зарядового и полевого откликов результаты выписаны в табл. 2. Размеры элементов из четырех соединений составляли $20 \times 20 \times 8.5 \mathrm{~mm}$ (длина, ширина, высота). Размеры соединения № 4
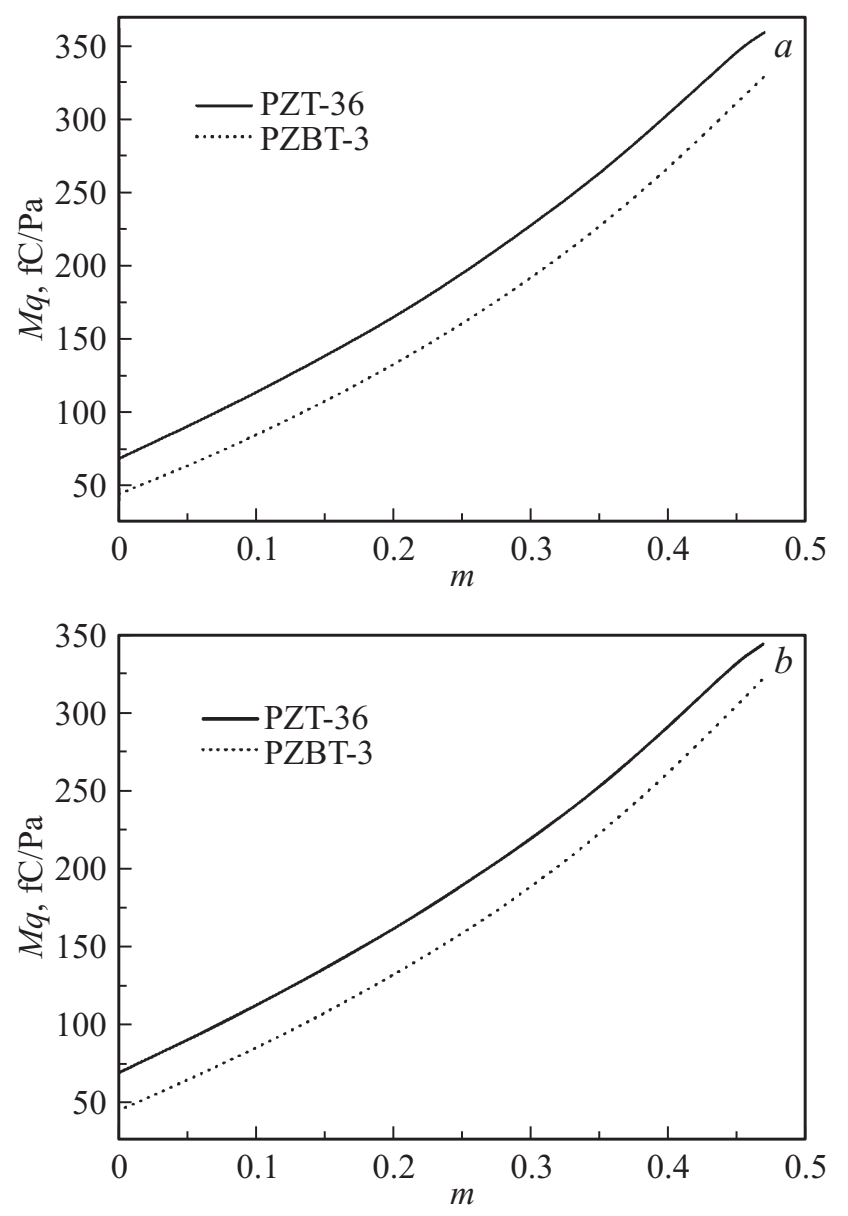

Рис. 1. Теоретическая зависимость зарядового отклика пористых композитов 0-3 на основе системы ЦТС от концентрации пор на частотах $20 \mathrm{~Hz}(a)$ и $1000 \mathrm{~Hz}(b)$. Данные для керамического компонента приведены в [7]. Геометрические размеры обоих составов предполагались равными $40 \times 40 \times 8.5 \mathrm{~mm}$. 
Таблица 2. Пьезоэлектрические и полевые свойства пористых композитов 0-3 на основе системы ЦТС. Теория и эксперимент

\begin{tabular}{|c|c|c|}
\hline $\begin{array}{c}\text { ЦТС-36 со степенью } \\
\text { пористости } 37 \%\end{array}$ & $\begin{array}{c}M_{q}, f \mathrm{C} / \mathrm{Pa} \\
(20 / 63 / 125) \mathrm{Hz}\end{array}$ & $\begin{array}{c}M_{u}, \mu \mathrm{V} / P a \\
(20 / 63 / 125 \mathrm{~Hz})\end{array}$ \\
\hline Теория & $70 / 67 / 66$ & $333 / 348 / 349$ \\
\hline 1 & $79 / 78 / 77$ & $315 / 316 / 314$ \\
\hline 2 & $84 / 78 / 79$ & $318 / 310 / 317$ \\
\hline 3 & $81 / 78 / 77$ & $360 / 350 / 360$ \\
\hline 4 & $76 / 74 / 73$ & $314 / 307 / 312$ \\
\hline 5 & $78 / 76 / 76$ & $342 / 328 / 332$ \\
\hline 6 & $74 / 72 / 71$ & $306 / 299 / 305$ \\
\hline 7 & $75 / 72 / 74$ & $299 / 292 / 301$ \\
\hline $\begin{array}{c}\text { ЦТС-36 со степенью } \\
\text { пористости } 40 \%\end{array}$ & $\begin{array}{c}M_{q}, f \mathrm{C} / \mathrm{Pa} \\
(20 / 63 / 125) \mathrm{Hz}\end{array}$ & $\begin{array}{c}M_{u}, \mu \mathrm{V} / P a \\
(20 / 63 / 125 \mathrm{~Hz})\end{array}$ \\
\hline Теория & $76 / 73 / 72$ & $407 / 425 / 427$ \\
\hline 1 & $89 / 86 / 85$ & $395 / 388 / 398$ \\
\hline 2 & $81 / 79 / 78$ & $372 / 365 / 374$ \\
\hline 3 & $93 / 91 / 90$ & $418 / 412 / 420$ \\
\hline 4 & $93 / 92 / 91$ & $411 / 421 / 424$ \\
\hline 5 & $91 / 88 / 87$ & $408 / 399 / 410$ \\
\hline 6 & $91 / 90 / 90$ & $399 / 393 / 402$ \\
\hline 7 & $92 / 90 / 89$ & $420 / 416 / 422$ \\
\hline 8 & $83 / 81 / 80$ & $364 / 360 / 370$ \\
\hline $\begin{array}{c}\text { ЦТБС-3 со степенью } \\
\text { пористости } 39 \%\end{array}$ & $\begin{array}{c}M_{q}, f \mathrm{C} / \mathrm{Pa} \\
(20 / 63 / 125) \mathrm{Hz}\end{array}$ & $\begin{array}{c}M_{u}, \mu \mathrm{V} / P a \\
(20 / 63 / 125 \mathrm{~Hz})\end{array}$ \\
\hline Теория & $67 / 66 / 65$ & $208 / 211 / 206$ \\
\hline 1 & $68 / 68 / 65$ & $213 / 217 / 211$ \\
\hline 2 & $67 / 66 / 64$ & $211 / 210 / 200$ \\
\hline 3 & $67 / 66 / 65$ & $208 / 208 / 208$ \\
\hline 4 & $67 / 65 / 64$ & $204 / 201 / 210$ \\
\hline 5 & $66 / 65 / 64$ & $205 / 220 / 201$ \\
\hline Теория (размер равен $40 \times 40 \times 8.5$ ) & $268 / 255 / 236$ & $199 / 214 / 200$ \\
\hline $6($ размер равен $40 \times 40 \times 8.5)$ & $242 / 232 / 229$ & $196 / 205 / 194$ \\
\hline 7 (размер равен $40 \times 40 \times 8.5)$ & $261 / 255 / 249$ & $195 / 217 / 205$ \\
\hline $8($ размер равен $40 \times 40 \times 8.5)$ & $251 / 244 / 231$ & $207 / 219 / 200$ \\
\hline $\begin{array}{c}\text { ЦТБС-3 со степенью } \\
\text { пористости } 44 \%\end{array}$ & $\begin{array}{c}M_{q}, f \mathrm{C} / \mathrm{Pa} \\
(20 / 63 / 125) \mathrm{Hz}\end{array}$ & $\begin{array}{c}M_{u}, \mu \mathrm{V} / P a \\
(20 / 63 / 125 \mathrm{~Hz})\end{array}$ \\
\hline Теория & $74 / 73 / 72$ & $250 / 251 / 254$ \\
\hline 1 & $76 / 74 / 73$ & $256 / 255 / 256$ \\
\hline 2 & $78 / 77 / 77$ & $264 / 264 / 266$ \\
\hline 3 & $80 / 79 / 78$ & $268 / 270 / 275$ \\
\hline 5 & $81 / 80 / 79$ & $269 / 268 / 277$ \\
\hline 6 & $62 / 61 / 60$ & $209 / 208 / 211$ \\
\hline
\end{tabular}

варьировали и кроме указанных размеров также составляли $40 \times 40 \times 8.5 \mathrm{~mm}$, что усиливало зарядовый отклик данного состава пропорционально величине площади образца. Разными последовательными номерами в табл. 2 показаны различные изделия из одного и того же состава для описания статистики эксперимента. В табл. 1 соответствующие данные для диэлектрических свойств были усреднены. 
Измерения зарядового $\left(M_{q}\right)$ и полевого $\left(M_{u}\right)$ откликов проводились на частотах 20, 63 и $125 \mathrm{~Hz}$, емкость образцов измерялась аналогично на данных частотах, а также на $1000 \mathrm{~Hz}$. Для диэлектрических исследований были выбраны несколько различных объектов каждого состава, что позволило методом усреднения определить оптимальные диэлектрические характеристики. Наибольшие статистические отклонения ДП между максимальными и минимальными значениями в пределах одной партии не превышали 15-20\%, что позволяет судить о надежности экспериментальной процедуры.

На рис. 1 изображены теоретические зависимости зарядового отклика $M_{q}$ от концентрации пористого компонента для рассматриваемых в работе составов на частотах 20 и $1000 \mathrm{~Hz}$ соответственно. Из анализа рис. 1 видно, что с ростом пористости зарядовый отклик резко растет во всем частотном диапазоне внешнего механического воздействия в пределах максвелл-вагнеровской релаксационной области. Расчеты при $m>0.47$ затруднены ввиду наличия при $m=0.5$ перколяционного перехода типа „жесткость-податливость“ [6], который приводит к обращению в бесконечность эффективных упругих податливостей системы [6] и, следовательно, невозможности корректной работы программы для подсчета $M_{q}$ и $M_{u}$.

\section{Нахождение функции распределения времен релаксации}

Другой важной и во многом нерешенной проблемой остается вопрос о распределении времен релаксации в гетерофазных керамиках и композитах $[11,12]$. До настоящего исследования подобные данные по пористым пьезоэлектрическим составам практически отсутствовали. В настоящей работе нами численно с помощью метода циклической подгонки Иманиши $[11,12]$ впервые получены функции распределения времен релаксации пористых композитов 0-3 системы ЦТС. Комплексная ДП произвольного диэлектрика, обладающего достаточно широким спектром времен релаксации в общем случае может быть записана следующим образом:

$$
\varepsilon^{*}=\varepsilon_{\infty}+\left(\varepsilon_{0}-\varepsilon_{\infty}\right) \int_{0}^{\infty} \frac{f(\tau)}{1+i \omega \tau} d \tau
$$

Здесь $\tau$ - это время релаксации, $f(\tau)$ в (2) - это функция распределения времен релаксации, определяемая в настоящей работе для пористых композитов $0-3$. Значением $\varepsilon_{0}$ являлась ДП, измеренная на частоте $20 \mathrm{~Hz}$ (левый край частотной зависимости диэлектрических спектров настоящей работы), а $\varepsilon_{\infty}$ есть ДП на частоте $1000 \mathrm{~Hz}$ (правый край релаксационной области). Листинг программы для расчетов в математической среде Matematica 5.0 приведен в Приложении. Для пяти рассматриваемых в настоящей работе составов соответствующие функции распределения времен релаксации $[11,12,15]$ получились очень сходными по

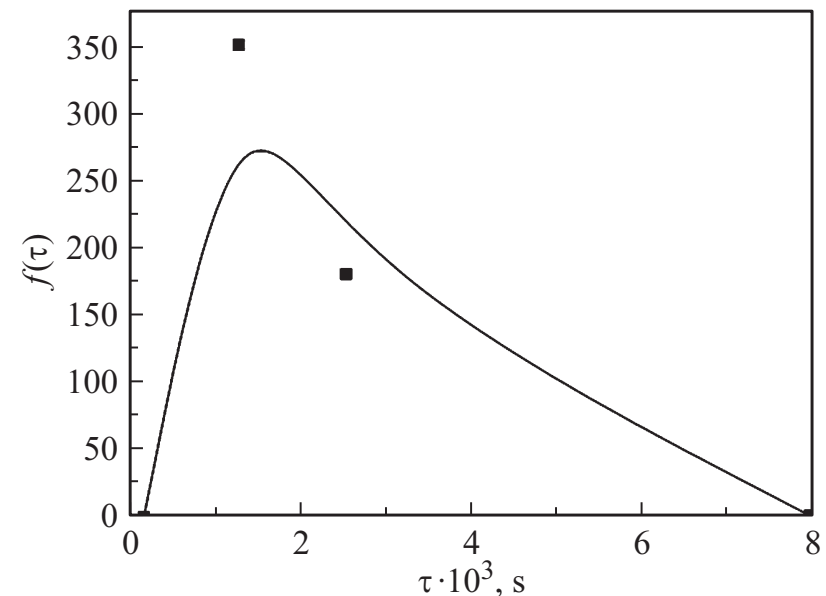

Рис. 2. Функция распределения времен межкомпонентной релаксации макроскопического заряда для диэлектрического спектра состава ЦТБС-3, определенная по четырем экспериментальным точкам 20,63, 125 и $1000 \mathrm{~Hz}$.

форме зависимости, имеют тип распределения КоулаКоула [15] и обладают ярко выраженным максимумом при $\tau \approx 1.5 \cdot 10^{-3} \mathrm{~s}$. На рис. 2 приведена функция распределения для состава № 5 по классификации, приведенной выше. Соединение точек сглаженной линиейсплайном и выполнение условия нормировки позволяет судить о правильности соответствующей аппроксимации. Монотонность частотной зависимости реальной части ДП, по которой осуществлялась подгонка, также говорит о том, что четырех точек вполне достаточно для построения $f(\tau)$ и отсутствия других максимумов. Программа в Приложении после ее исполнения вычисляет в третьем столбце значение функции распределения времен релаксации (переменная Ual) в зависимости от $\tau$. В четвертом столбце выдается достигнутая разность между реальными и подгоночными значениями. Чем она меньше, тем большая точность подгонки достигнута. Нами достигалась точность порядка нескольких единиц при абсолютных значениях ДП порядка тысячи. В конце вычисляется нормировка (полный интеграл от функции распределения, условие нормировки есть $\left.\int_{0}^{\infty} f(\tau) d \tau=1\right)$, которая должна быть равна единице, что надо каждый раз проверять после нахождения $f(\tau)$. Так как условие нормировки на рис. 2 выполняется полностью, можно сказать, что в настоящей работе на частотной зависимости ДП полностью пройдена вся ее релаксационная область. Второй столбец дает время релаксации (аргумент функции $f(\tau)$ ), а последний пятый есть начальное отклонение теории от экспериментальных значений. Остальные параметры программы Приложения будут понятны специалисту по данной теме $[11,12,15]$ после изучения настоящей работы и [11,12]. Данная программа может быть легко дополнена до сколь угодно большого числа экспериментальных точек в зависимости от конкретного вида диэлектрического или пьезоэлектрического спектра. 


\section{Приложение}

Листинг программы для подгонки спектров по четырем значениям в среде Matematica 5.0

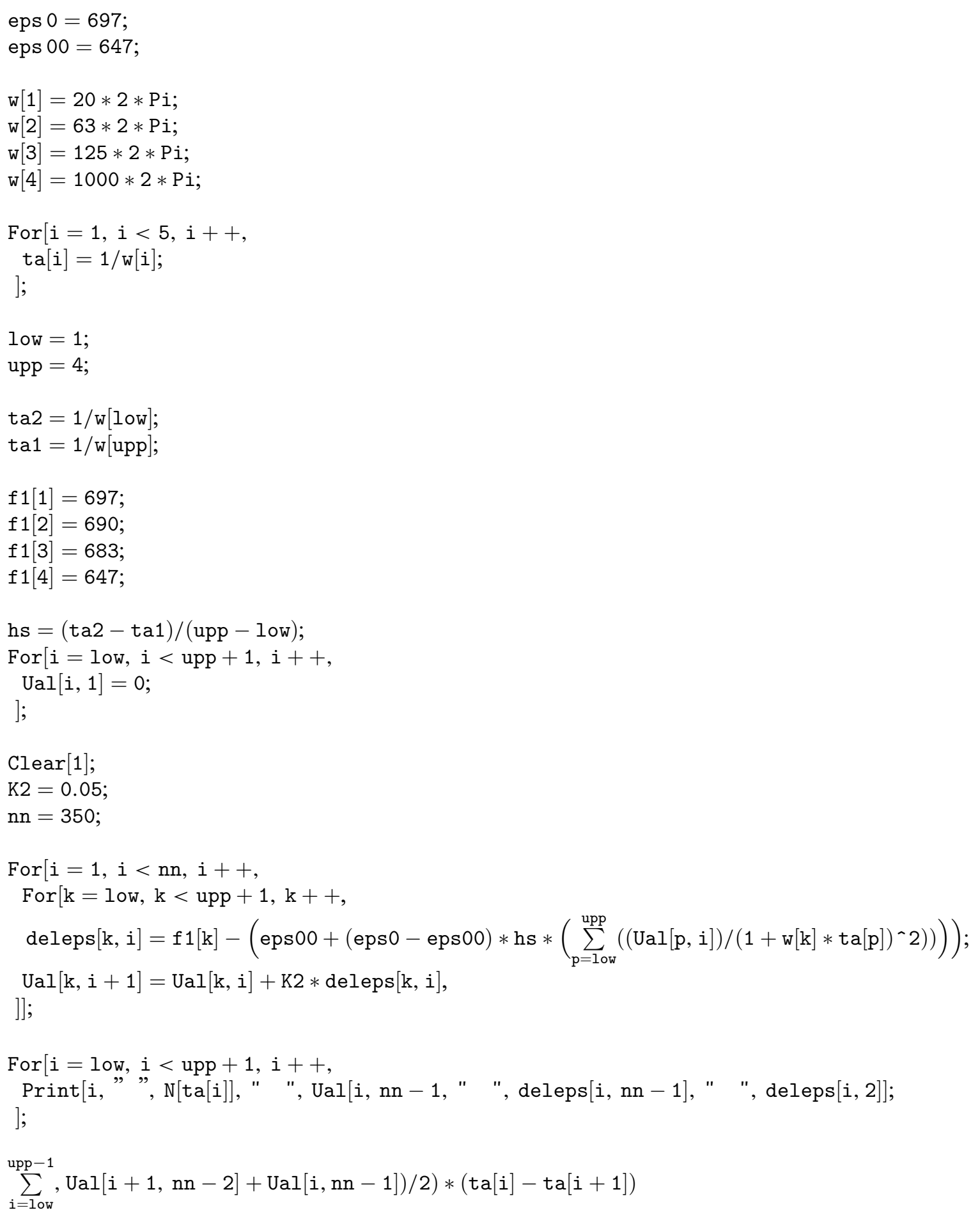




\section{Заключение}

Теоретические значения зарядового отклика для всех рассмотренных составов очень близки к эксперименту. Дальнейшее понижение частоты при построении теоретических зависимостей ведет к резкому росту зарядового отклика, что связано со значительным накоплением максвелл-вагнеровского заряда на поверхностях раздела „керамика-пора“. Приложение как механических, так и электрических низкочастотных полей приводит к межкомпонентной релаксации заряда и существованию релаксационной области с резким изменением всех эффективных параметров с частотой. Несколько худшее совпадение теоретических значений емкости с экспериментом может быть вызвано эффектом пьезоэлектрического зажатия на границах пор (так называемый „сlamping effect“ [1]). Эффективная емкость в частотном интервале от 20 до $100 \mathrm{~Hz}$ зависит от частоты достаточно слабо, однако при росте частоты до $1000 \mathrm{~Hz}$ она резко падает, что подтверждается экспериментально. Это находит отражение в определенной на рис. 2 функции распределения времен релаксации. Частотный полевой отклик $M_{u}$ с ростом частоты иногда испытывает не релаксацию, а ретардацию $[13,14]$, что проявляется в его более выраженном отклике на высоких частотах. В некоторых случаях полевой отклик $M_{u}$ немонотонен с изменением частоты, что подтверждается теоретическими расчетами. Для теоретического описания неупорядоченной среды, которой и является пористая керамика, перспективен метод эффективной среды, который позволяет прогнозировать частотные зависимости эффективных констант при введении в рассмотрение комплексной проницаемости. Пористые композиты в силу сложности своей внутренней структуры должны описываться моделями типа $[2-6,8-10]$, которые являются статистическими и не отдают симметрийное предпочтение компонентам композитного материала. Функция распределения времен релаксации имеет самое вероятное время (явный максимум) и коул-коуловский характер общей зависимости [15]. Уменьшение частоты внешнего электрического или механического воздействия сопровождается значительной релаксацией заряда, ретардацией полевого отклика и усилением ДП и пьезосвойств во всех рассмотренных нами составах независимо от степени пористости, что является важным для широкого практического применения. Полученные результаты могут также применяться для описания магнитоэлектрических и магнитных систем на низких частотах [16] с целью получения магнитоэлектрического усиления эффективных констант и их релаксации. Полное описание функции распределения времен релаксации возможно при дискретизированных диэлектрических спектрах [12], которые обязательно должны охватывать всю частотную область релаксационного процесса. Результаты, полученные в настоящей работе, находятся в хорошей корреляции с недавними теоретическими и экспериментальными исследованиями по пористой керамике [17].

\section{Благодарности}

Исследования проведены при финансовой поддержке Министерства образования и науки РФ в рамках реализации комплексного проекта „Разработка и создание высокотехнологичного производства мобильного гидроакустического комплекса освещения обстановки в различных акваториях Мирового океана на основе современных пьезоэлектрических средств нового поколения“ (Договор № 03.G25.31.0276 от 29.05.2017).

Работа выполнена с использованием оборудования ЦКП „Высокие технологии““ ЮФУ.

Авторы выражают благодарность кандидату химических наук, доценту кафедры общей и неорганической химии ЮФУ С.Н. Свирской за постоянный интерес к работе и ценные обсуждения.

\section{Список литературы}

[1] Turik A.V., Radchenko G.S. // J. Phys. D: Appl. Phys. 2002. Vol. 35. P. 1188-1192.

[2] Турик А.В., Чернобабов А.И., Радченко Г.С., Турик С.А. // ФТТ. 2004. Т. 46. Вып. 12. С. 2139-2142.

[3] Мэзон У. Физическая акустика. М.: Изда-во Мир, 1966. $589 \mathrm{c}$.

[4] Ландау Л.Д., Лившии Е.М. Электродинамика сплошных сред. М.: Физматлит, 2005. $651 \mathrm{c.}$

[5] Aleshin V. // J. Appl. Phys. 2000. Vol. 88. P. 3587-3591.

[6] Алешин В.И., Цихочкий Е.С., Яценко В.К. // ЖТФ. 2004. Т. 74. Вып. 1. С. 62-67.

[7] Отраслевой стандарт „Материалы пьезокерамические“ 11.0444.1987. 148 с.

[8] Eshelby J.D. The Continual Theory of Dislocations. Moscow. 1963. $247 \mathrm{p}$.

[9] Dunn M.L., Wienecke H.A. // Int. J. Sol. Struct. 1997. Vol. 34. P. 3571-3582.

[10] Huang J.H., Kuo W.-S. // Acta Mater. 1996. Vol. 44. P. 4889 4898.

[11] Imanishi Y., Adachi K., Kotaka T. // J. Chem. Phys. 1988. Vol. 89. P. 7593-7598.

[12] Беляев Б.А., Дрокин Н.А., Шабанов В.Ф. // ФТТ. 2005. Т. 47. Вып. 9. С. 1722-1726.

[13] Турик А.В., Радченко Г.С. // ФТТ. 2003. Т. 45. Вып. 6. C. 1013-1016.

[14] Радиенко Г.С., Турик А.В. // ФТТ. 2003. Т. 45. Вып. 9. C. 1676-1679.

[15] Турик А.В., Радченко Г.С., Чернобабов А.И., Турик С.А., Супрунов В.В. // ФТТ. 2006. Т. 48. Вып. 6. С. 1088-1090.

[16] Radchenko G.S. // J. Appl Phys. A. 2012. Vol. 109. N 2. P. 449-457.

[17] Рыбянеи А.Н., Константинов Г.М., Науменко А.А., Швецова Н.А., Макарьев Д.И., Луговая М.А. // ФТТ. 2015. Т. 57.Вып. 3. С. $515-518$. 